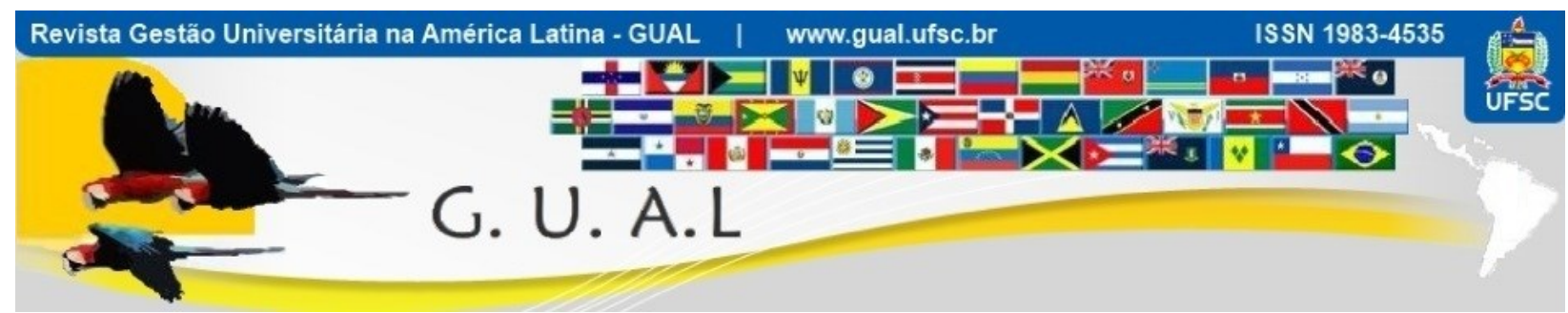

DOI: http://dx.doi.org/10.5007/1983-4535.2014v7n1p63

\title{
EXPANSÃO DA EDUCAÇÃO SUPERIOR NO BRASIL A PARTIR DO REUNI: O CURSO NOTURNO DE ODONTOLOGIA DA UNIVERSIDADE FEDERAL DO RIO GRANDE DO SUL
}

\author{
EXPANSION OF HIGHER EDUCATION IN BRAZIL FROM THE 'REUNI' \\ EXPERIENCE: EVENING COURSE OF DENTISTRY IN THE FEDERAL \\ UNIVERSITY OF RIO GRANDE DO SUL
}

Juliana Maciel de Souza, Mestranda Universidade Federal do Rio Grande do Sul - UFRGS juli.desouza@ufrgs.br

Helena Corrêa Weschenfelder, Graduanda Universidade Federal do Rio Grande do Sul - UFRGS helenawes@gmail.com

Ramona Fernanda Ceriotti Toassi, Doutora Universidade Federal do Rio Grande do Sul - UFRGS ramona.fernanda@ufrgs.br

Recebido em 03/junho/2013

Aprovado em 17/novembro/2013

Sistema de Avaliação: Double Blind Review

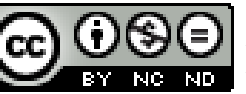

Esta obra está sob uma Licença Creative Commons Atribuição-Uso. 


\title{
RESUMO
}

Este artigo é resultado de um estudo que tem por objetivo analisar o perfil do estudante ingressante do curso noturno de Odontologia em uma universidade pública no sul do Brasil, bem como os motivos de escolha, expectativas em relação à profissão e perspectivas de atuação profissional. Iniciado no segundo semestre de 2010, o curso noturno de Odontologia da Universidade Federal do Rio Grande do Sul propõe-se a inserir o estudante que trabalha durante o dia na universidade. Trata-se de um estudo observacional transversal. Foram convidados a participar da pesquisa todos os estudantes que realizaram a matrícula no curso em 2010, 2011 e 2012 (n=90). A coleta de dados foi realizada por meio da aplicação de um questionário semiestruturado. O estudo foi aprovação pelo Comitê de Ética em Pesquisa da Universidade (protocolo $\mathrm{n}^{\circ} 21797$ ). Um total de 88 estudantes participaram desse estudo (taxa de resposta $=97,8 \%$ ). A maior parte dos estudantes são mulheres, jovens, brancos, solteiros, sem filhos, com até dois irmãos, naturais do estado do Rio Grande do Sul, residem com os pais e trabalham. É necessário o acompanhamento permanente da experiência dos cursos criados com base na proposta 'REUNI' em cada instituição federal.

Palavras-chave: Odontologia. Educação em Odontologia. Estudantes de Odontologia. Ensino Superior. Escolha da Profissão.

\begin{abstract}
This article is the result of a study that aimed to analyze the profile of first-year dental students - evening course - at a public university in the south of Brazil. It was also evaluated the reasons for pursuing dentistry as an occupation, professional expectations and perspectives. Having started in the second half of 2010, the evening course is proposed to insert a 'diurnal worker' student in the university. A cross-sectional study was performed using data from a self-administered questionnaire to all first-year dental students (along 2010, 2011 and 2012) at the Federal University of Rio Grande do Sul, Brazil $(\mathrm{n}=90)$. The study was approved by the Ethics in Research Committee of the University (protocol $\mathrm{n}^{\circ} 21797$ ). A total of 88 students participated in this study (response rate $=97.8$ percent). Most students are women, young, white, unmarried, no children, up to two brothers, natural state of Rio Grande do Sul, reside with their parents and are currently employed. It is necessary to constantly monitor the experience of the courses created based on the 'REUNI' government proposition at each federal facility.
\end{abstract}

Keywords: Dentistry. Dental Education. Students, Dental. Education, Highter. Career Choice. 


\section{INTRODUÇÃO}

A ampliação do acesso à universidade não é só uma preocupação governamental, mas da sociedade contemporânea, principalmente no que se refere ao aumento de vagas que permita a equidade e a formação de qualidade, com ênfase na responsabilidade social (NEVES; RAIZER; FACHINETTO, 2007).

O sistema de educação do Brasil conta com 2365 instituições de ensino superior, sendo que apenas 4,3\% dessas são instituições federais (INSTITUTO NACIONAL DE ESTUDOS E PESQUISAS EDUCACIONAIS ANÍSIO TEIXEIRA, 2013), as quais concentram o maior número de pesquisadores e pesquisas, com corpo docente composto na sua maioria por doutores, oferecendo ensino, pesquisa e extensão de maior qualidade no país (COSTA; COSTA; BARBOSA, 2013).

Com a proposta de expansão e reestruturação das universidades públicas apresentada pelo governo federal por meio do Programa de Apoio a Planos de Reestruturação e Expansão das Universidades Federais (REUNI), as universidades federais do Brasil passaram por intensas transformações. Uma das dimensões do REUNI apontou a ampliação de vagas para ingresso na universidade, especialmente no período noturno (BRASIL, 2007).

Esse cenário de mudanças no ensino superior do Brasil também incluiu a Universidade Federal do Rio Grande do Sul (UFRGS). Com a oportunidade de expansão a partir do REUNI, no segundo semestre de 2010, a Faculdade de Odontologia da UFRGS (FO/UFRGS) propôs a criação do primeiro curso em período noturno do estado do Rio Grande do Sul com a oferta de 30 vagas anuais. A primeira turma ingressou na universidade no segundo semestre de 2010. A intenção principal é atender a demanda de quem desenvolve sua atividade profissional durante o dia e dispõe somente do horário noturno para realizar sua formação acadêmica.

A organização curricular do curso noturno teve como base o projeto pedagógico do curso de Odontologia já existente na Instituição (período integral) e os preceitos do REUNI. As atividades que compõem o curso são desenvolvidas no turno da noite e o período indicado de integralização é de 16 semestres (UNIVERSIDADE FEDERAL DO RIO GRANDE DO SUL, 2010).

Diante da necessidade de verificar se o objetivo de inserir o estudante trabalhador na universidade está sendo contemplado e considerando a importância de se conhecer as características do grupo de estudantes que acessa o curso, a presente pesquisa buscou analisar 
o perfil sociodemográfico do estudante ingressante no curso noturno de Odontologia da UFRGS, bem como os motivos dessa escolha, expectativas em relação à profissão e perspectivas de atuação profissional.

Além de caracterizar o estudante do curso noturno de Odontologia, o conhecimento do perfil dos ingressantes pode servir como instrumento de planejamento das ações tanto do corpo docente do curso, quanto da equipe de trabalho que nele atua.

\section{METODOLOGIA}

O estudo caracteriza-se como observacional transversal. Foi realizado na Faculdade de Odontologia na Universidade Federal do Rio Grande do Sul.

Todos os estudantes que realizaram a matrícula no curso noturno de graduação em Odontologia da UFRGS em 2010, 2011 e 2012 foram convidados a participar do estudo como voluntários. Excluíram-se os estudantes que não concordaram em assinar o termo de consentimento livre e esclarecido ou que estavam ausentes no momento da realização da pesquisa.

A coleta de dados aconteceu por meio da aplicação de um questionário semiestruturado, pré-testado, contendo questões relacionadas ao perfil sociodemográfico dos estudantes e a opção pela Odontologia (motivos, expectativas e perspectivas de atuação profissional).

Para a análise dos dados sociodemográficos dos estudantes e das respostas referentes às questões fechadas do questionário, foi criado um banco de dados com as informações coletadas, digitadas no software estatístico Statistical Package for Social Sciences (SPSS), versão 18 para Windows. Já as respostas das questões abertas foram analisadas e interpretadas seguindo o método da análise de conteúdo (BARDIN, 2011).

O estudo teve aprovação do Comitê de Ética em Pesquisa da Universidade (protocolo $\left.\mathrm{n}^{\mathrm{o}} 21797\right)$.

\section{RESULTADOS}

Os resultados do estudo são apresentados em dois momentos: perfil sociodemográfico dos estudantes; opção pela Odontologia (motivos, expectativas e perspectivas de atuação profissional). 


\subsection{PERFIL SOCIODEMOGRÁFICO DOS ESTUDANTES}

Dos 90 ingressantes do curso noturno da FO/UFRGS - turmas de 2010, 2011 e 2012 88 participaram do estudo (taxa de resposta de 97,8\%). Desses estudantes, a maior parte são mulheres (67,1\%), jovens (60,2\% tinham de 17 a 22 anos de idade), brancos $(85,2 \%)$, solteiros $(85,2 \%)$, sem filhos $(69,3 \%)$, com até dois irmãos $(60,3 \%)$ e naturais do estado do Rio Grande do Sul (89,7\%) (Tabela 1).

Tabela 1 Características demográficas dos estudantes do curso noturno de Odontologia.

\begin{tabular}{|c|c|c|c|c|c|c|c|c|}
\hline \multirow[b]{2}{*}{ VARIÁVEIS } & \multicolumn{2}{|c|}{2010} & \multicolumn{2}{|c|}{2011} & \multicolumn{2}{|c|}{2012} & \multicolumn{2}{|c|}{ TOTAL } \\
\hline & $\mathbf{n}$ & $(\%)$ & $\mathbf{n}$ & $(\%)$ & $\mathbf{n}$ & $(\%)$ & $\mathbf{n}$ & $(\%)$ \\
\hline \multicolumn{9}{|l|}{ SEXO } \\
\hline Feminino & 19 & $(65,5)$ & 22 & $(73,3)$ & 18 & $(62,1)$ & 59 & $(67,1)$ \\
\hline Masculino & 9 & $(31,0)$ & 8 & $(26,7)$ & 11 & $(37,9)$ & 28 & $(31,8)$ \\
\hline Não informou & 1 & $(3,5)$ & -- & $(--)$ & -- & $(--)$ & 1 & $(1,1)$ \\
\hline \multicolumn{9}{|l|}{ IDADE } \\
\hline 17-19 anos & 8 & $(27,60)$ & 7 & $(23,4)$ & 7 & $(24,2)$ & 22 & $(25,0)$ \\
\hline 20-22 anos & 6 & $(20,7)$ & 15 & $(50,0)$ & 10 & $(34,4)$ & 31 & $(35,2)$ \\
\hline 23-25 anos & 2 & $(7,0)$ & 4 & $(13,3)$ & 5 & $(17,2)$ & 11 & $(12,5)$ \\
\hline 26-29 anos & 4 & $(13,8)$ & 2 & $(6,7)$ & 3 & $(10,4)$ & 9 & $(10,2)$ \\
\hline 30-32 anos & 4 & $(13,8)$ & 1 & $(3,3)$ & 2 & $(7,0)$ & 7 & $(8,0)$ \\
\hline $36-38$ anos & -- & $(--)$ & 1 & $(3,3)$ & 1 & $(3,4)$ & 2 & $(2,3)$ \\
\hline 39-41 anos & 3 & $(10,3)$ & -- & $(--)$ & -- & $(--)$ & 3 & $(3,4)$ \\
\hline $44-51$ anos & 1 & $(3,4)$ & -- & $(--)$ & 1 & $(3,4)$ & 2 & $(2,2)$ \\
\hline Não informou & 1 & $(3,4)$ & -- & $(--)$ & -- & $(--)$ & 1 & $(1,2)$ \\
\hline \multicolumn{9}{|l|}{ ETNIA AUTOREFERIDA } \\
\hline Branco & 26 & $(89,7)$ & 23 & $(76,6)$ & 26 & $(89,7)$ & 75 & $(85,2)$ \\
\hline Negro & 1 & $(3,4)$ & 2 & $(6,7)$ & 2 & $(6,9)$ & 5 & $(5,7)$ \\
\hline Pardo & 1 & $(3,4)$ & 5 & $(16,7)$ & 1 & $(3,4)$ & 7 & $(8,0)$ \\
\hline Não informou & 1 & $(3,5)$ & -- & $(--)$ & -- & $(--)$ & 1 & $(1,1)$ \\
\hline \multicolumn{9}{|l|}{ ESTADO CIVIL } \\
\hline Solteiro & 23 & $(79,3)$ & 27 & $(90,0)$ & 25 & $(86,2)$ & 75 & $(85,2)$ \\
\hline Casado/com companheiro & 6 & $(20,7)$ & 3 & $(10,0)$ & 4 & $(13,8)$ & 13 & $(14,8)$ \\
\hline \multicolumn{9}{|l|}{ FILHOS } \\
\hline Um filho & 3 & $(10,3)$ & -- & $(--)$ & 1 & $(3,4)$ & 4 & $(4,5)$ \\
\hline Dois filhos & 1 & $(3,4)$ & -- & $(-)$ & 2 & $(6,9)$ & 3 & $(3,4)$ \\
\hline Três filhos & 2 & $(6,9)$ & -- & $(--)$ & -- & $(--)$ & 2 & $(2,3)$ \\
\hline Não tem filhos & 5 & $(17,3)$ & 30 & $(100,0)$ & 26 & $(89,7)$ & 61 & $(69,3)$ \\
\hline Não informou & 18 & $(62,1)$ & -- & $(--)$ & -- & $(--)$ & 18 & $(20,5)$ \\
\hline \multicolumn{9}{|l|}{ IRMÃOS } \\
\hline Um a dois irmãos & 16 & $(55,2)$ & 20 & $(66,7)$ & 17 & $(58,7)$ & 53 & $(60,3)$ \\
\hline Três irmãos & 5 & $(17,2)$ & 4 & $(13,3)$ & 3 & $(10,3)$ & 12 & $(13,6)$ \\
\hline Quatro a cinco irmãos & 2 & $(6,9)$ & 3 & $(10,0)$ & 2 & $(6,9)$ & 7 & $(8,0)$ \\
\hline Oito irmãos & -- & $(--)$ & -- & $(--)$ & 1 & $(3,4)$ & 1 & $(1,1)$ \\
\hline Não tem irmãos & 4 & $(13,8)$ & 3 & $(10,0)$ & 5 & $(17,3)$ & 12 & $(13,6)$ \\
\hline Não informou & 2 & $(6,9)$ & -- & $(--)$ & 1 & $(3,4)$ & 3 & $(3,4)$ \\
\hline \multicolumn{9}{|l|}{ ESTADO DE ORIGEM } \\
\hline Rio Grande do Sul & 28 & $(96,6)$ & 23 & $(76,7)$ & 28 & $(96,6)$ & 79 & $(89,7)$ \\
\hline Paraná & -- & $(--)$ & 1 & $(3,3)$ & -- & $(--)$ & 1 & $(1,1)$ \\
\hline Tocantins & -- & $(--)$ & 1 & $(3,3)$ & -- & $(--)$ & 1 & $(1,1)$ \\
\hline Rio de Janeiro & -- & $(--)$ & 1 & $(3,3)$ & -- & $(--)$ & 1 & $(1,1)$ \\
\hline São Paulo & -- & $(--)$ & 1 & $(3,3)$ & -- & $(--)$ & 1 & $(1,2)$ \\
\hline Mato Grosso & 1 & $(3,4)$ & -- & $(--)$ & -- & $(--)$ & 1 & $(1,2)$ \\
\hline
\end{tabular}




\begin{tabular}{ccccccccc} 
Mato Grosso do Sul & -- & $(--)$ & 1 & $(3,3)$ & -- & $(--)$ & 1 & $(1,2)$ \\
Não informou & -- & $(--)$ & 2 & $(6,8)$ & 1 & $(3,4)$ & 3 & $(3,4)$ \\
\hline TOTAL & $\mathbf{2 9}$ & $\mathbf{( 1 0 0 , 0 )}$ & $\mathbf{3 0}$ & $\mathbf{( 1 0 0 , 0 )}$ & $\mathbf{2 9}$ & $\mathbf{( 1 0 0 , 0 )}$ & $\mathbf{8 8}$ & $\mathbf{( 1 0 0 , 0 )}$ \\
\hline
\end{tabular}

Com relação à formação no ensino fundamental e médio, respectivamente, $39,7 \%$ e $46,5 \%$ dos ingressantes cursaram exclusivamente em escola pública. Esses estudantes não foram os primeiros da família a cursar o ensino superior $(77,3 \%)$, frequentaram algum curso pré-vestibular $(73,8 \%)$ e realizaram até 2 vestibulares antes de ingressar no curso de Odontologia da UFRGS (55\%). Tiveram o intervalo entre o final do ensino médio e o início do ensino superior de 3 anos ou mais $(62,5 \%)$ e não precisaram mudar de cidade para estudar Odontologia $(69,3 \%)$.

Os estudantes residem com os pais $(56,9 \%)$, trabalham $(55,7 \%)$, possuem renda mensal de 1 a 3 salários mínimos (40,9\%) e carga horária semanal de trabalho entre 30 e 40 horas $(34,1 \%)$. Dos 49 estudantes que trabalham, apenas 5 relataram ser os principais responsáveis pelo sustento da família (Tabela 2).

Tabela 2 Características socioeconômicas dos estudantes do curso noturno de Odontologia.

\begin{tabular}{|c|c|c|c|c|c|c|c|c|}
\hline \multirow[b]{2}{*}{ VARIÁVEIS } & \multicolumn{2}{|c|}{2010} & \multicolumn{2}{|c|}{2011} & \multicolumn{2}{|c|}{2012} & \multicolumn{2}{|c|}{ TOTAL } \\
\hline & $\mathbf{n}$ & $(\%)$ & $\mathbf{n}$ & $(\%)$ & $\mathbf{n}$ & $(\%)$ & $\mathbf{n}$ & $(\%)$ \\
\hline \multicolumn{9}{|l|}{ COM QUEM RESIDE } \\
\hline Com os pais & 17 & $(58,7)$ & 19 & $(63,3)$ & 14 & $(48,3)$ & 50 & $(56,9)$ \\
\hline Com outros parentes & 1 & $(3,4)$ & 5 & $(16,7)$ & 3 & $(10,3)$ & 9 & $(10,2)$ \\
\hline Com os pais e outros parentes & -- & $(--)$ & -- & $(--)$ & 1 & $(3,4)$ & 1 & $(1,1)$ \\
\hline Sozinho (a) & 3 & $(10,4)$ & 2 & $(6,7)$ & 2 & $(6,9)$ & 7 & $(8,0)$ \\
\hline Com amigos & 1 & $(3,4)$ & 1 & $(3,3)$ & 2 & $(6,9)$ & 4 & $(4,5)$ \\
\hline Em casa de estudante & -- & $(--)$ & -- & $(--)$ & 1 & $(3,4)$ & 1 & $(1,1)$ \\
\hline Com companheiro (a) & 2 & $(6,9)$ & 2 & $(6,7)$ & 3 & $(10,3)$ & 7 & $(8,0)$ \\
\hline Com os filhos & 1 & $(3,4)$ & -- & $(--)$ & -- & $(--)$ & 1 & $(1,1)$ \\
\hline Com pais e companheiro (a) & -- & $(--)$ & -- & $(--)$ & 1 & $(3,5)$ & 1 & $(1,1)$ \\
\hline Com companheiro (a) e filhos & 3 & $(10,4)$ & -- & $(--)$ & 1 & $(3,5)$ & 4 & $(4,5)$ \\
\hline Em uma pensão & -- & $(--)$ & 1 & $(3,3)$ & -- & $(--)$ & 1 & $(1,2)$ \\
\hline Não informou & 1 & $(3,4)$ & -- & $(--)$ & 1 & $(3,5)$ & 2 & $(2,3)$ \\
\hline $\begin{array}{l}\text { ATIVIDADE PROFISSIONAL } \\
\text { Não trabalha e os gastos são financiados pela } \\
\text { família }\end{array}$ & 12 & $(41,4)$ & 12 & $(40,0)$ & 11 & $(38,0)$ & 35 & $(39,8)$ \\
\hline Trabalha e recebe ajuda da família & 5 & $(17,3)$ & 11 & $(36,7)$ & 9 & $(31,1)$ & 25 & $(28,4)$ \\
\hline Trabalha e se sustenta & 2 & $(6,9)$ & 6 & $(20,0)$ & 3 & $(10,3)$ & 11 & $(12,5)$ \\
\hline Trabalha e contribui com o sustento da família & 4 & $(13,8)$ & 1 & $(3,3)$ & 3 & $(10,3)$ & 8 & $(9,1)$ \\
\hline $\begin{array}{l}\text { Trabalha e é o principal responsável pelo } \\
\text { sustento da família }\end{array}$ & & $(10,3)$ & -- & $(--)$ & 2 & $(6,9)$ & 5 & $(5,7)$ \\
\hline Não informou & 3 & $(10,3)$ & -- & $(--)$ & 1 & $(3,4)$ & 4 & $(4,5)$ \\
\hline \multicolumn{9}{|l|}{ RENDA PESSOAL* } \\
\hline Até 1 salário mínimo & 2 & $(6,9)$ & -- & $(--)$ & 5 & $(17,3)$ & 7 & $(8,0)$ \\
\hline 1 a 3 salários mínimos & 10 & $(34,5)$ & 15 & $(50,0)$ & 11 & $(37,8)$ & 36 & $(40,9)$ \\
\hline 4 a 5 salários mínimos & 1 & $(3,4)$ & 3 & $(10,0)$ & -- & $(--)$ & 4 & $(4,6)$ \\
\hline 6 a 8 salários mínimos & 2 & $(6,8)$ & -- & $(--)$ & -- & $(--)$ & 2 & $(2,2)$ \\
\hline Não informou & 2 & $(6,9)$ & -- & $(--)$ & 2 & $(6,9)$ & 4 & $(4,5)$ \\
\hline Não trabalha & 12 & $(41,5)$ & 12 & $(40,0)$ & 11 & $(38,0)$ & 35 & $(39,8)$ \\
\hline
\end{tabular}




\section{CARGA HORÁRIA DE TRABALHO}

SEMANAL

$10-20$ horas

25 horas

$30-40$ horas

$44-50$ horas

Não informou

Não trabalha

TOTAL

\begin{tabular}{cccccccc}
2 & $(6,9)$ & 2 & $(6,7)$ & 2 & $(6,9)$ & 6 & $(6,8)$ \\
-- & $(--)$ & 1 & $(3,3)$ & -- & $(--)$ & 1 & $(1,1)$ \\
13 & $(44,7)$ & 9 & $(30,0)$ & 8 & $(27,6)$ & 30 & $(34,1)$ \\
-- & $(--)$ & 5 & $(16,7)$ & 5 & $(17,3)$ & 10 & $(11,4)$ \\
2 & $(6,9)$ & 1 & $(3,3)$ & 3 & $(10,3)$ & 6 & $(6,8)$ \\
12 & $(41,5)$ & 12 & $(40,0)$ & 11 & $(37,9)$ & 35 & $(39,8)$ \\
$\mathbf{2 9}$ & $\mathbf{( 1 0 0 , 0 )}$ & $\mathbf{3 0}$ & $\mathbf{( 1 0 0 , 0 )}$ & $\mathbf{2 9}$ & $\mathbf{( 1 0 0 , 0 )}$ & $\mathbf{8 8}$ & $\mathbf{( 1 0 0 , 0 )}$ \\
\hline
\end{tabular}

*Valor do salário mínimo quando a aplicação dos questionários: $\mathrm{R} \$ 510,00$ (2010), R\$ 545,00 (2011) e R\$ 622,00 (2012).

Em relação à atuação profissional, $16,9 \%$ dos estudantes que trabalham possuem vínculo com a saúde, atuando nas seguintes áreas: fonoaudiologia, saúde pública, instrumentação cirúrgica, farmácia/bioquímica e odontologia (auxiliar de consultório odontológico e prótese dentária).

Sobre a família dos estudantes, $39,8 \%$ dos pais e $35,2 \%$ das mães possuem ensino médio completo e $33 \%$ dos pais e $36,3 \%$ das mães, ensino superior completo. A maior parte dos pais e das mães trabalha e a renda familiar mensal para $45,4 \%$ dos estudantes foi de 1 a 5 salários mínimos e de 6 a 10 salários mínimos para 26,1\%. A presença de dentista na família foi relatada apenas por $23,9 \%$ dos estudantes do curso noturno, sendo mais observada entre pais ou irmãos e com tios e/ou primos.

\subsection{SOBRE A OPÇÃO PELA ODONTOLOGIA: MOTIVOS, EXPECTATIVAS E PERSPECTIVAS DE ATUAÇÃO PROFISSIONAL}

Pouco mais da metade dos estudantes (53,4\%) não ingressou em outro curso de graduação antes de iniciar a Odontologia e, dos que ingressaram, a maioria não o concluiu. Os estudantes sentem-se completamente seguros $(51,1 \%)$ ou seguros $(30,7 \%)$ quanto à escolha pela Odontologia (Tabela 3).

Tabela 3 Ingresso/área em curso de graduação anterior à Odontologia e segurança quanto à escolha pelo curso.

\begin{tabular}{lllllllll}
\hline & $\mathbf{2 0 1 0}$ & & $\mathbf{2 0 1 1}$ & & $\mathbf{2 0 1 2}$ & \multicolumn{3}{c}{ TOTAL } \\
VARIÁVEIS & $\mathbf{n}$ & $\mathbf{( \% )}$ & $\mathbf{n}$ & $\mathbf{( \% )}$ & $\mathbf{n}$ & $\mathbf{( \% )}$ & $\mathbf{n}$ & $\mathbf{( \% )}$ \\
\hline INGRESSO EM CURSO SUPERIOR ANTERIOR & & & & & & & & \\
Não & 14 & $(48,3)$ & 17 & $(56,6)$ & 16 & $(55,2)$ & 47 & $(53,4)$ \\
Sim, mas abandonou & 6 & $(20,7)$ & 6 & $(20,1)$ & 8 & $(27,6)$ & 20 & $(22,7)$ \\
Sim e está cursando & 2 & $(6,9)$ & 2 & $(6,7)$ & 4 & $(13,8)$ & 8 & $(9,1)$ \\
Sim, já concluiu & 6 & $(20,7)$ & 4 & $(13,3)$ & -- & $(--)$ & 10 & $(11,4)$ \\
Não informou & 1 & $(3,4)$ & 1 & $(3,3)$ & 1 & $(3,4)$ & 3 & $(3,4)$ \\
ÁREA DO CURSO ANTERIOR FREQUENTADO & & & & & & & & \\
Ciências da saúde & 4 & $(13,8)$ & 5 & $(16,7)$ & 3 & $(10,3)$ & 12 & $(13,5)$ \\
Ciências humanas & 3 & $(10,3)$ & 1 & $(3,4)$ & -- & $(--)$ & 4 & $(4,5)$
\end{tabular}


Ciências biológicas

Ciências sociais aplicadas

Ciências exatas e da terra

Engenharias

Ciências sociais aplicadas

Ciências agrárias

Linguística, letras e artes

Ciências da saúde e ciências sociais aplicadas

Ciências da saúde e linguística, letras e artes

Ciências sociais aplicadas e linguística, letras e artes

Não frequentou ensino superior anteriormente

Não informou

\section{SEGURANÇA QUANTO À ESCOLHA PELA} ODONTOLOGIA

Completamente seguro

Seguro

Indiferente

Inseguro

Completamente inseguro

Não respondeu

TOTAL

$\begin{array}{llllllll}1 & (3,4) & 1 & (3,3) & -- & (--) & 2 & (2,3) \\ 1 & (3,4) & 1 & (3,3) & -- & (--) & 2 & (2,3) \\ 1 & (3,4) & 1 & (3,4) & -- & (--) & 2 & (2,3) \\ -- & (--) & -- & (--) & 2 & (6,9) & 2 & (2,3) \\ 3 & (10,4) & 2 & (6,6) & 3 & (10,4) & 8 & (8,9) \\ 1 & (3,5) & -- & (--) & 1 & (3,5) & 2 & (2,3) \\ -- & (--) & 1 & (3,3) & -- & (--) & 1 & (1,2) \\ -- & (--) & -- & (--) & 1 & (3,4) & 1 & (1,2) \\ -- & (--) & -- & (--) & 1 & (3,4) & 1 & (1,2) \\ -- & (--) & -- & (--) & 1 & (3,4) & 1 & (1,2) \\ 14 & (48,3) & 17 & (56,6) & 16 & (55,2) & 47 & (53,4) \\ 1 & (3,5) & 1 & (3,4) & 1 & (3,5) & 3 & (3,4)\end{array}$
(100,0)

Quando questionados sobre os motivos pelos quais optaram pela Odontologia, os estudantes mencionaram a 'realização pessoal e profissional', pela 'segurança, posição social, conforto financeiro' e pelo 'interesse em atuar na comunidade'.

Já sobre as expectativas com a profissão, a 'realização profissional', a 'realização pessoal/de um sonho/vocação' e a 'formação qualificada', foram as mais relatadas pelos estudantes.

No momento do ingresso no curso, a pretensão de atuação profissional aliando serviço público e privado foi, de modo geral, a escolha mais observada, especialmente nos estudantes da turma de 2012 (Tabela 4).

Tabela 4 Pretensão de atuação profissional dos estudantes do curso noturno de Odontologia.

\begin{tabular}{lllllllll}
\hline & $\mathbf{2 0 1 0}$ & \multicolumn{2}{c}{$\mathbf{2 0 1 1}$} & \multicolumn{2}{c}{$\mathbf{2 0 1 2}$} & \multicolumn{2}{c}{ TOTAL } \\
PRETENSÃO DE ATUAÇÃO PROFISSIONAL & $\mathbf{n}$ & $\mathbf{( \% )}$ & $\mathbf{n}$ & $\mathbf{( \% )}$ & $\mathbf{n}$ & $\mathbf{( \% )}$ & $\mathbf{n}$ & $\mathbf{( \% )}$ \\
\hline Serviço público e privado & 4 & $(13,8)$ & 4 & $(13,4)$ & 14 & $(48,3)$ & 22 & $(25,0)$ \\
Consultório particular/clínica privada & 4 & $(13,8)$ & 5 & $(16,7)$ & 1 & $(3,4)$ & 10 & $(11,4)$ \\
Serviço público, privado e universidade & 1 & $(3,4)$ & -- & $(--)$ & 7 & $(24,2)$ & 8 & $(9,2)$ \\
Somente em serviço público & 4 & $(13,8)$ & 3 & $(10,0)$ & -- & $(--)$ & 7 & $(8,0)$ \\
Universidade (docência/pesquisa) & -- & $(--)$ & 1 & $(3,3)$ & 2 & $(6,9)$ & 3 & $(3,4)$ \\
Serviço privado e universidade & -- & $(--)$ & -- & $(--)$ & 2 & $(6,9)$ & 2 & $(2,2)$ \\
Serviço público e universidade & -- & $(--)$ & -- & $(--)$ & 2 & $(6,9)$ & 2 & $(2,2)$ \\
Hospital & -- & $(--)$ & 1 & $(3,3)$ & -- & $(--)$ & 1 & $(1,1)$ \\
Não informou/ Não sabe informar & 16 & $(55,2)$ & 16 & $(53,3)$ & 1 & $(3,4)$ & 33 & $(37,5)$ \\
\hline TOTAL & $\mathbf{2 9}$ & $\mathbf{( 1 0 0 , 0 )}$ & $\mathbf{3 0}$ & $\mathbf{( 1 0 0 , 0 )}$ & $\mathbf{2 9}$ & $\mathbf{( 1 0 0 , 0 )}$ & $\mathbf{8 8}$ & $\mathbf{( 1 0 0 , 0 )}$ \\
\hline
\end{tabular}




\section{DISCUSSÃO}

Historicamente as instituições de ensino superior caracterizaram-se pela formação tradicional de caráter diurno e rigidez seriada, constituindo-se como espaços ocupados por uma minoria que não possuía vínculo com o mundo do trabalho (ARROYO, 1991).

Com a expansão de vagas para o ingresso em universidades públicas do Brasil, especialmente no período da noite (BRASIL, 2007), o ensino noturno passa a protagonizar as discussões sobre modelos de ensino, buscando uma maior pluralidade institucional e reconhecimento do direito universal ao conhecimento, à cultura e à qualificação.

No contexto brasileiro, o estudante de curso noturno em sua maioria, é aquele que trabalha durante o dia e busca a formação profissional no curso de graduação à noite, agregando conhecimento, vivência pessoal e relacionamento com colegas e professores, de forma que possa encontrar melhores condições de inserção no mercado de trabalho (TERRIBILI FILHO, 2008).

Avaliando a literatura em relação ao perfil do estudante dos cursos de Odontologia no Brasil, as evidências encontradas mostram a predominância de estudos relacionados aos cursos diurnos, em período integral de formação (TOASSI et al., 2011; FREIRE et al., 2011; CAVALCANTI et al., 2010; COSTA et al., 2010; YAMAUCHI; MOURA; PERES, 2008; REZENDE et al., 2007; BRUSTOLIN et al., 2006; UNFER et al., 2004; SLAVUTZKY et al., 2002; FREIRE; SOUZA; PEREIRA, 1995; BOTTI; SANTOS, 1986; ALMEIDA JÚNIOR et al., 1983; ARBENZ et al., 1973). Poucos estudos identificam o perfil do estudante de cursos noturnos de Odontologia (LOFFREDO et al., 2004; JUNQUEIRA et al., 2002; FREITAS; NAKAYAMA, 1995).

No Rio Grande do Sul, o primeiro curso de Odontologia no período noturno iniciou suas atividades em 2010 na Universidade Federal do Rio Grande do Sul (UFRGS), com a oferta de 30 vagas anuais. Tal ampliação do acesso ao ensino superior em Odontologia pretendeu oportunizar um espaço qualificado de formação acadêmica para pessoas que desenvolvem suas atividades profissionais durante o dia e dispõe somente do horário noturno para realizar seus estudos.

Em três anos de acompanhamento do curso noturno, foi verificado um importante predomínio de mulheres, confirmando o processo de feminização das profissões na área da saúde no Brasil e, de modo especial na Odontologia, ao longo dos anos (TOASSI et al., 2011; FREIRE et al., 2011; COSTA; DURÃES; ABREU, 2010; YAMAUCHI; MOURA; PERES, 
2008; REZENDE et al., 2007; BRUSTOLIN et al., 2006; LOFFREDO et al., 2004; UNFER et al., 2004; JUNQUEIRA et al., 2002; CARVALHO; CARVALHO; SAMPAIO, 1997; FREITAS; NAKAYAMA, 1995; FREIRE; SOUZA; PEREIRA, 1995; BOTTI; SANTOS, 1986; ALMEIDA JUNIOR et al., 1983). Este alto percentual de mulheres na Odontologia também pode ser percebido no contexto internacional (AL-BITAR; SONBO; AL-OMARI, 2008; HALLISSEY; HANNIGAN; RAY, 2000).

Pesquisa sobre o perfil atual e tendências do cirurgião-dentista brasileiro mostrou que as mulheres cirurgiãs-dentistas com inscrição principal ativa são a maioria em 25 dos 27 estados do Brasil. Essa predominância de mulheres na Odontologia pode ser observada desde o final dos anos 90. A profissão, atualmente, tem maioria feminina (56,3\%), o que acompanha o ingresso progressivo das mulheres brasileiras no ensino superior, principalmente, a partir dos anos 80 (MORITA; HADDAD; ARAÚJO, 2010).

O fenômeno crescente da 'feminização' é destacado como uma das seis tendências do mercado de trabalho em saúde, junto com a expansão da capacidade instalada, a municipalização dos empregos, a ambulatorização dos atendimentos, a maior qualificação da equipe e a flexibilidade dos vínculos. Dados do Censo de 2000 do Instituto Brasileiro de Geografia e Estatística (IBGE) e do Ministério da Educação (MEC) revelaram a tendência deste processo de feminização: entre os profissionais com diploma universitário, $61 \%$ são mulheres. Dessas, cerca de 95\% são nutricionistas, 90\% enfermeiras, 51\% cirurgiãs-dentistas e 36\% médicas (MACHADO; OLIVEIRA; MOYSÉS, 2010).

A idade desses estudantes da noite variou de 17 a 51 anos, mas a maior parte tinha de 17 a 22 anos, o que se aproxima dos resultados encontrados em estudos sobre o perfil de estudantes em cursos noturnos de Odontologia (LOFFREDO et al., 2004; JUNQUEIRA et al., 2002; FREITAS; NAKAYAMA, 1995) e diurnos/período integral (TOASSI et al., 2011; CAVALCANTI et al., 2010; HAWLEY et al., 2008; REZENDE et al., 2007; BRUSTOLIN et al., 2006; UNFER et al., 2004; CARVALHO; CARVALHO; SAMPAIO, 1997; FREIRE; SOUZA; PEREIRA, 1995; BOTTI; SANTOS, 1986; ALMEIDA JUNIOR et al., 1983).

Outras características observadas nos estudantes do curso da noite também se aproximam de resultados encontrados na literatura com estudantes de Odontologia, cursos diurnos/período integral, como: serem solteiros (TOASSI et al., 2011; CAVALCANTI et al., 2010; REZENDE et al., 2007; BRUSTOLIN et al., 2006; UNFER et al., 2004; LOFFREDO et al., 2004; JUNQUERIA et al., 2002; CARVALHO; CARVALHO; SAMPAIO, 1997; 
FREIRE; SOUZA; PEREIRA, 1995), sem filhos (TOASSI et al., 2011; FREIRE; SOUZA; PEREIRA, 1995; BRUSTOLIN et al., 2006), naturais do mesmo estado da universidade que frequentam (TOASSI etal., 2011; BRUSTOLIN et al., 2006; YAMAUCHI; MOURA; PERES, 2008; CAVALCANTI et al., 2010) e terem feito curso pré-vestibular antes do ingresso na universidade (TOASSI et al., 2011; YAMAUCHI; MOURA; PERES, 2008; BRUSTOLIN et al., 2006; LOFFREDO et al., 2004; FREIRE; SOUZA; PEREIRA, 1995).

No que se referiu à formação no ensino fundamental e médio, a maior parte desses estudantes o realizaram em escola pública, dado encontrado também em estudantes do curso noturno de Odontologia em Araraquara, São Paulo (LOFFREDO et al., 2004). Já nos cursos diurnos/período integral, os estudantes apresentaram, preferencialmente, formação no ensino fundamental e médio realizada em escolas privadas (TOASSI et al., 2011; CAVALCANTI et al., 2010; YAMAUCHI; MOURA; PERES, 2008; BRUSTOLIN et al., 2006; CARVALHO; CARVALHO; SAMPAIO, 1997; FREIRE; SOUZA; PEREIRA, 1995).

Pouco mais da metade dos estudantes do curso noturno relatou não ter ingressado em outro curso superior antes do ingresso na Faculdade de Odontologia da UFRGS. Esse percentual aumenta para mais de $80 \%$ entre os estudantes de Odontologia matriculados no curso de período integral na mesma instituição (TOASSI et al., 2011).

Tal diferença também pode ser observada quando analisadas as variáveis relacionadas à família dos estudantes. No curso em período integral (TOASSI et al., 2011), identificou-se que mais da metade dos pais dos estudantes possuíam ensino superior completo (pais e mães), enquanto que os do curso noturno apresentavam maior percentual para o ensino médio completo ou ensino superior completo.

A renda familiar para a maior parte dos estudantes foi de 1 a 5 salários mínimos, semelhante ao encontrado por Cavalcanti et al. (2010), no curso diurno de Odontologia da Universidade Estadual da Paraíba. Este valor, porém, foi inferior ao encontrado em outras pesquisas com estudantes de cursos diurnos/período integral (TOASSI et al., 2011; HAWLEY; DITMYER; SANDOVAL, 2008; BRUSTOLIN et al., 2006; CARVALHO; CARVALHO; SAMPAIO, 1997; FREIRE; SOUZA; PEREIRA, 1995) e noturnos (LOFFREDO et al., 2004; FREITAS; NAKAYAMA, 1995).

Um aspecto a ser destacado refere-se à situação dos estudantes do curso noturno quanto à inserção no mercado de trabalho. Mais da metade desses estudantes trabalha $(55,7 \%)$, diferentemente do que foi encontrado por Loffredo et al. (2004), em que somente 
$11,5 \%$ dos estudantes de Odontologia noturno possuíam rendimento financeiro próprio. Pesquisas realizadas em cursos diurnos/período integral de Odontologia no Brasil mostram um predomínio de estudantes que se dedicam exclusivamente à vida acadêmica e que não trabalham (TOASSI et al., 2011; CAVALCANTI et al., 2010; JUNQUEIRA et al., 2002; ALMEIDA JÚNIOR et al., 1983).

O projeto pedagógico do curso noturno de Odontologia da UFRGS foi elaborado de forma a atender o objetivo de inserir o estudante, trabalhador na Universidade, de forma que todas as atividades de ensino obrigatórias são oferecidas exclusivamente à noite. Desta forma, possibilita ao estudante trabalhador prosseguir com suas atividades profissionais durante o dia e dedicar-se à vida acadêmica à noite, obtendo assim sua graduação na área da saúde (UNIVERSIDADE FEDERAL DO RIO GRANDE DO SUL, 2010).

Outro aspecto que chama a atenção nesse estudo é que apenas 10,2\% dos estudantes que trabalham são os principais responsáveis pelo sustento da família, enquanto $51 \%$ trabalha, mas recebe ajuda da família. Terribili Filho (2008) caracteriza este estudante como o 'estudante-trabalhador', ou seja, é o estudante que dedica uma grande carga horária semanal ao trabalho, porém possui a vida financeira parcialmente mantida pela família. Para este estudante, o planejamento do futuro profissional será determinado pela obtenção do diploma de ensino superior.

A maior parte dos estudantes do curso noturno de Odontologia da UFRGS estava completamente seguro em relação à escolha do curso. Os motivos de opção pelo curso mais citados foram a realização pessoal e profissional, seguido pela segurança/ tranquilidade no futuro/posição social/conforto financeiro e interesse em atuar em comunidade. Esses motivos também foram observados em outras pesquisas realizadas com estudantes de Odontologia (CAVALCANTI et al., 2010, AL-BITAR; SONBOL; AL-OMARI, 2008, BRUSTOLIN et al., 2006, CARVALHO; CARVALHO; SAMPAIO, 1997, FREIRE; SOUZA; PEREIRA, 1995).

Apenas um estudante relatou ter escolhido a Odontologia por ser uma profissão liberal. Resultado diferente foi encontrado nos estudos de Hawley, Ditmyer e Sandoval (2008), Rezende et al. (2007), Slavutzky et al. (2002), Junqueira et al.(2002), Hallissey, Hannigan e Ray (2000) e Arbenz et al. (1973), em que este foi um dos motivos mais citados.

Destaca-se que, ainda que se trate de um curso oferecido integralmente no período da noite, apenas dois estudantes referiram tê-lo escolhido por este motivo. Já Loffredo et al. 
(2004) verificaram que 27\% dos estudantes de Odontologia de Araraquara - UNESP, curso noturno, escolheram o curso pela possibilidade de ser realizado à noite e desta maneira terem mais tempo livre durante o dia.

Em relação às expectativas com a Odontologia, as respostas mais citadas foram realização profissional, realização pessoal/ de um sonho/ vocação e formação qualificada. Essas mesmas expectativas foram encontradas em estudos realizados com estudantes de Odontologia do curso diurno da mesma Universidade (TOASSI et al., 2011; SLAVUTZKY et al., 2002).

Após o término do curso, um número importante de estudantes (cerca de 38\%) ainda não sabe informar sobre onde pretende atuar. Dos que informaram a pretensão de atuação profissional, a maior parte afirmou a intenção de aliar o trabalho no serviço público ao serviço privado. Esse resultado assemelha-se ao observado recentemente nos estudantes do curso diurno de Odontologia da UFRGS (TOASSI et al., 2011) e diverge do resultado encontrado em estudo anterior à reestruturação curricular nesse mesmo curso (SLAVUTZKY et al., 2002), em que predominou a intenção dos estudantes de atuarem exclusivamente no setor privado (consultório próprio/compartilhado ou clínica própria/compartilhada).

As mudanças curriculares na Faculdade de Odontologia da UFRGS (UNIVERSIDADE FEDERAL DO RIO GRANDE DO SUL, 2005), baseadas nas Diretrizes Curriculares Nacionais (BRASIL, 2002), e o espaço importante que a Odontologia tem ocupado nas políticas públicas de saúde do país, podem justificar as diferentes percepções observadas nesses estudantes ao longo do tempo e potencializar a formação de um dentista preparado para atuar no Sistema Único de Saúde e comprometido com o cuidado efetivo levando em conta a realidade social do Brasil.

\section{CONSIDERAÇÕES FINAIS}

A ampliação do acesso ao ensino superior e a formação de qualidade são questões centrais das políticas educacionais da última década (NEVES; RAIZER; FACHINETTO, 2007). Foi neste contexto que a UFRGS propôs a implementação de um curso noturno de Odontologia, com a intenção de inserir o estudante trabalhador na universidade, comprometendo-se com a formação acadêmica que possibilite a esse estudante uma preparação para enfrentar os desafios da vida profissional e voltada para a promoção da qualidade de vida da população. 
Os resultados deste estudo demonstraram que o curso noturno de Odontologia segue a tendência de feminização observada nas profissões da saúde, com estudantes jovens, brancos, solteiros, sem filhos, com até dois irmãos, naturais do estado do Rio Grande do Sul, que residem com os pais e que estão completamente seguros quanto a sua opção profissional. Pouco mais da metade desses estudantes trabalha, demonstrando a importância de um curso noturno para a inserção do estudante trabalhador no ensino superior na área da saúde. A motivação predominante de escolha pela Odontologia foi a realização pessoal e profissional e há a perspectiva de aliar o serviço público e privado na atuação profissional.

É preciso o acompanhamento permanente dos estudantes ao longo do curso e também da experiência concreta de ampliação de vagas e cursos vinculados ao projeto REUNI nas universidades federais do país, buscando verificar se os objetivos estão sendo alcançados.

\section{REFERÊNCIAS}

AL-BITAR, Z. B.; SONBOL, H. N.; AL-OMARI, I. K. Reasons for choosing dentistry career by Arab dental students. Eur J Dent Educ, England, v. 12, no.4, p. 247-251, Oct. 2008.

ALMEIDA JUNIOR, E. et al. A escolha da profissão odontológica. Motivação consciente. Rev. fac. odontol. Univ. Fed. Bahia., Salvador, v.3, jan./dez. 1983.

ARBENZ, G. O. et al. Motivos conscientes na escolha da profissão odontológica. Rev. Fac. Odontol. Univ. São Paulo., São Paulo, v. 11, n.1, p.101-110, 1973.

ARROYO, M. G. A universidade, o trabalhador e o curso noturno. Universidade e Sociedade, Brasília, ano 1, v. 1, p. 25-32, fev. 1991.

BARDIN, L. Análise de conteúdo. São Paulo: Edições 70, 2011.

BOTTI, M. R. V.; SANTOS, G. M. C. Perspectiva do exercício profissional na odontologia. RGO (Porto Alegre)., Porto Alegre, v. 34, n. 2, p. 155-159, mar./abr. 1986.

BRASIL. Conselho Nacional de Educação. Câmara de Educação Superior. Resolução CNE/CNS 3/2002, de 19 de fevereiro de 2002. Institui as Diretrizes Curriculares Nacionais do Curso de Graduação em Odontologia. Diário Oficial da União, Brasília, 4 de março de 2002. Seção 1, p. 10.

BRASIL. Ministério da Educação. REUNI Reestruturação e Expansão das Universidades Federais: diretrizes gerais. Brasília, 2007.

BRUSTOLIN, J. et al. Perfil do acadêmico de odontologia da Universidade do Planalto Catarinense - Lages- SC, Brasil. Rev. ABENO., Brasília, v. 6, n.n1, p. 70-76, 2006. 
CARVALHO, D. R.; CARVALHO, A. C. P; SAMPAIO, H. Motivações e expectativas para o curso e para o exercício da odontologia. Rev. reg. Aracatuba assoc. paul. cir. Dent., Aracatuba, v. 51, n. 4, jul./ago. 1997.

CAVALCANTI, A. L. et al. Motivos de ingresso e de evasão dos acadêmicos de odontologia de uma instituição pública. Rev. odontol. UNESP., Araraquara, v. 39, n. 2, p. 95-99, mar./abr. 2010.

COSTA, D. M.; COSTA, A. M.; BARBOSA, F. V. Financiamento público e expansão da educação superior federal no Brasil: o REUNI e as perspectivas para o REUNI 2. Revista GUAL, Florianópolis, v. 6, n. 1, p. 106-127, 2013.

COSTA, S. M. et al. Motivos de escolha da odontologia: vocação, opção ou necessidade? Arq. odontol., Belo Horizonte, v. 46, n. 1, p. 28-37, jan./mar. 2010.

COSTA, S. M.; DURÃES, S. J. A.; ABREU, M. H. N. G. Feminização do curso de odontologia da Universidade Estadual de Montes Claros. Ciênc. saúde coletiva., Rio de Janeiro, v. 15, supl. 1, p. 1865-1873, 2010.

FREIRE, M. C. M.; SOUZA, C. S.; PEREIRA, H. R. O perfil do acadêmico de odontologia da universidade de Goiás. Divulgação em Saúde para Debate, Rio de Janeiro, n.10, p. 1520, jun. 1995.

FREIRE, M. C. M. et al. Motivation towards career choice of brazilian freshman students in a fifteen-year period. Eur J Dent Educ, England, v. 75, no 1, p. 115-121, Jan. 2011.

FREITAS, S. F. T.; NAKAYAMA, M.Y. Um perfil do estudante de odontologia no estado de São Paulo. Divulgação em Saúde para Debate, Rio de Janeiro, n. 10, p. 29-37, jun. 1995.

HALLISSEY, J.; HANNIGAN, A.; RAY, N. Reasons for choosing dentistry as a career - a survey of dental students attending a dental school in Ireland during 1998-99. Eur J Dent Educ, England, v. 4, no. 2, p. 77-81, May 2000.

HAWLEY, N. J.; DITMYER, M. M.; SANDOVAL, V. A. Predental students' attitudes toward and perceptions of the dental profession. Eur J Dent Educ, England, v. 72, no.12, p. 1458-1464, Dec. 2008.

INSTITUTO NACIONAL DE ESTUDOS E PESQUISAS EDUCACIONAIS ANÍSIO TEIXEIRA. Censo da educação superior: 2011 resumo técnico. Brasília: Instituto Nacional de Estudos e Pesquisas Educacionais Anísio Teixeira, 2013.114 p. Disponível em: $<$ www.ufrgs.br/sai/dados-resultados/avaliacao-das-ies-em-geral/arquivos-avaliacao-iesgeral/resumo tecnico censo educacao superior_2011.pdf $>$. Acesso em: 03 jun. 2013.

JUNQUEIRA, J. C. et al. Quem é e o que pensa o graduando de odontologia. Rev. odontol. UNESP., São Paulo, v. 31, n.2, p. 269-284, 2002. 
LOFFREDO, L. C. M. et al. Característica socioeconômica, cultural e familiar de estudantes de odontologia. Rev. odontol. UNESP., Marilia, v.33, n.4 p. 175-182, 2004.

MACHADO, M. H.; OLIVEIRA, E. S.; MOYSÉS, N. M. N. Tendências do mercado de trabalho em saúde no Brasil. Conferência Internacional sobre Pesquisas em Recursos Humanos em Saúde. Rio de Janeiro, 2010.

MORITA, M. C.; HADDAD, A. E.; ARAÚJO, M. E. Perfil atual e tendências do cirurgiãodentista brasileiro. Maringá: Dental Press, 2010. 96 p.

NEVES, C. E. B.; RAIZER, L.; FACHINETTO, R. F. Acesso, expansão e equidade na educação superior: novos desafios para a política educacional brasileira. Sociologias, Porto Alegre, ano 9, n. 17, p. 124-157, jan./jun. 2007.

REZENDE, F. P. et al. Perfil, motivações e expectativas dos graduandos e graduados em odontologia. Rev. odontol. Univ. Cid. Sao Paulo., São Paulo, v.19, n. 2, p.165-72, maio/ago. 2007.

SLAVUTZKY, S. M. B. et al. Mercado de trabalho: perfil do acadêmico de odontologia da Universidade Federal do Rio Grande do Sul. Rev. Fac. Odontol. Porto Alegre., Porto Alegre, v. 43, n. 2, p. 3-6, dez. 2002.

TERRIBILI FILHO, A. Ensino superior noturno no Brasil: estudar para trabalhar ou trabalhar para estudar? Pensamento e Realidade., São Paulo, ano 11, n. 22, p. 43-65, 2008.

TOASSI, R. F. C. et al. Perfil sociodemográfico e perspectivas em relação à profissão do estudante de odontologia da Universidade Federal do Rio Grande do Sul, Brasil. Rev. Fac. Odontol. Porto Alegre., Porto Alegre, v. 52, n. 1/3, p. 25-32, jan./dez. 2011.

UNFER, B. et al. Expectativas dos acadêmicos de odontologia quanto a formação e futura profissão. Saúde, Santa Maria, v. 30, n.1-2, p.33-40, 2004.

UNIVERSIDADE FEDERAL DO RIO GRANDE DO SUL. Faculdade de Odontologia. Projeto Político Pedagógico. Porto Alegre, 2005. Disponível em:

$<$ http://www.ufrgs.br/odonto/projeto_pedagogico_odontologia_curso_diurno $>$. Acesso em: 03 jun. 2013.

UNIVERSIDADE FEDERAL DO RIO GRANDE DO SUL. Faculdade de Odontologia.

Projeto Político Pedagógico. Porto Alegre, 2010. Disponível em:

$<$ http://www.ufrgs.br/odonto/projeto_pedagogico_odontologia_curso_noturno $>$. Acesso em: 03 jun. 2013.

YAMAUCHI, S.; MOURA, P. G.; PERES, S. H. C. S. Análise do perfil educacional do desempenho acadêmico e da valorização à iniciação científica. Rev. ABENO., Brasília, v.8, n.2, p.167-173, 2008. 